\title{
Detection of genuine multipartite entanglement based on principal basis matrix representations
}

\author{
Hui Zhao, ${ }^{1}$ Yu-Qiu Liu, ${ }^{1}$ Shao-Ming Fei, ${ }^{2,3}$ Zhi-Xi Wang ${ }^{2}$ and Naihuan Jing ${ }^{4}$ \\ ${ }^{1}$ Department of Mathematics, Faculty of Science, Beijing University of Technology, Beijing 100124, China \\ ${ }^{2}$ School of Mathematical Sciences, Capital Normal University, Beijing 100048, China \\ ${ }^{3}$ Max-Planck-Institute for Mathematics in the Sciences, Leipzig 04103, Germany \\ ${ }^{4}$ Department of Mathematics, North Carolina State University, Raleigh, NC 27695, USA
}

\begin{abstract}
We study the genuine multipartite entanglement in tripartite quantum systems by using the principal basis matrix representations of density matrices. Using the Schmidt decomposition and local unitary transformation, we first convert the general states to simpler forms and then construct some special matrices from the correlation tensors of the simplified density matrices. Based on the different linear combinations of these matrices, necessary conditions are presented to detect genuine multipartite entanglement of tripartite states. Detailed examples show that our method can detect more entangled states than previous ones.
\end{abstract}

Keywords: Genuine multipartite entanglement, Principal basis, Correlation tensor PACS: 03.65.Ud, 02.10.Ox, 03.67.Mn

\section{Introduction}

Quantum entanglement is the key resource in many information processing tasks such as quantum cryptography [1], teleportation [2] and dense coding [3]. The genuine multipartite entanglement (GME) has particularly significant advantages such as in highly sensitive metrological tasks [4, 5]. It is of importance to study proper descriptions and effective detections of the genuine multipartite entanglement for given quantum states.

A lot of researches have been done towards the detection of entanglement and genuine multipartite entanglement [6, 7, 8]. The methods to detect k-nonseparability and k-partite entanglement by using of quantum Fisher information were proposed in [9]. By considering the basis dependence of the permutation operation, the authors in [10] obtained strong bounds on 
a multipartite nonseparability measure. The separability criteria to identify genuinely entangled and nonseparable n-partite mixed quantum states were derived in [11]. The results with experimentally implemented processes indicated that the compressive method works equally well with both entangled and product input states and output measurement resources even in the presence of noise [12]. The authors in [13] presented entanglement witness operators for detecting GME which require only two local measurement settings. A sufficient criterion for detecting GME was derived by using local sum uncertainty relations [14]. L. M. Yang et. al. [15] proposed a criterion for detecting genuine tripartite entanglement based on quantum Fisher information. Y. Akbari-Kourbolagh [16] found sufficient criteria for detecting the entanglement of three-qubit states in the vicinity of GHZ, W states and the PPT entangled states. Based on the Bloch representation of density matrices, several criteria to detect GME were also derived. In [17] the authors gave a sufficient condition for GME and a lower bound for the GME concurrence by using the norms of correlation tensor. M. Li et. al. [18] studied the separability of tripartite states by introducing an operator related to the transformations on the subsystem. In [19] the authors uncovered some separability criteria of bipartite and multipartite entanglement.

In this paper, we study the GME of tripartite quantum systems by using the principal basis matrix presentation of density matrices [20]. Consider the $d$-dimensional Hilbert spaces $H^{d}$ with orthonormal basis $\{|a\rangle\}_{a=0}^{d-1}$. Let $E_{i j}$ be the $d \times d$ matrix with the only nonzero entry 1 at the position $(i, j)$, and $\omega$ be a fixed $d$-th primitive root of unity. The principal basis matrices are given by $A_{i j}=\sum_{m \in Z_{d}} \omega^{i m} E_{m, m+j}$, where $\omega^{d}=1, i, j \in Z_{d}$ and $Z_{d}$ is $Z$ modulo $d$. It is readily apparent that $\operatorname{tr}\left(A_{i j} A_{k l}^{\dagger}\right)=\delta_{i k} \delta_{j l} d[21]$.

We use the principal basis matrices to expand a given density matrix. Then we employ Schmidt decomposition [22] and local unitary (LU) transformations 25] to transform the density matrix into a simpler form so as to construct useful invariants of GME. For any bipartite state $\rho$ in $H_{1}^{d_{1}} \otimes H_{2}^{d_{2}}, \rho$ can be expressed as $\rho=\frac{1}{d_{1} d_{2}}\left(I_{d_{1}} \otimes I_{d_{2}}+\sum_{(i, j)} a_{i j} A_{i j}^{(1)} \otimes I_{d_{2}}+\sum_{(i, j)} b_{i j} I_{d_{1}} \otimes A_{i j}^{(2)}+\right.$ $\left.\sum_{(i, j),(k, l)} c_{i j}^{k l} A_{i j}^{(1)} \otimes A_{k l}^{(2)}\right)$. Define the $\left(d_{1}^{2}-1\right) \times\left(d_{2}^{2}-1\right)$ matrix $S=\left[c_{i j}^{k l}\right]$. For example, when $\rho$ in 
$H_{1}^{2} \otimes H_{2}^{3}$

$$
S=\left[\begin{array}{cccccccc}
c_{01}^{01} & c_{01}^{02} & c_{01}^{10} & c_{01}^{11} & c_{01}^{12} & c_{01}^{20} & c_{01}^{21} & c_{01}^{22} \\
c_{10}^{01} & c_{10}^{02} & c_{10}^{10} & c_{10}^{11} & c_{10}^{12} & c_{10}^{20} & c_{10}^{21} & c_{10}^{22} \\
c_{11}^{01} & c_{11}^{02} & c_{11}^{10} & c_{11}^{11} & c_{11}^{12} & c_{11}^{20} & c_{11}^{21} & c_{11}^{22}
\end{array}\right]
$$

Denote $U A U^{\dagger}$ by $A^{U}$. Let $\rho^{\prime}=\rho^{\left(U_{1} \otimes U_{2}\right)}$, where $U_{1}$ and $U_{2}$ are unitary matrices. One sees that $\sum_{(i, j),(k, l)} c_{i j}^{k l}\left(A_{i j}^{(1)}\right)^{U_{1}} \otimes\left(A_{k l}^{(2)}\right)^{U_{2}}=\sum_{(i, j),(k, l)}\left(\sum_{\left(i^{\prime}, j^{\prime}\right),\left(k^{\prime}, l^{\prime}\right)} m_{i^{\prime} j^{\prime}}^{i j} c_{i^{\prime} j^{\prime}}^{k^{\prime} l^{\prime}} n_{k^{\prime} l^{\prime}}^{k l}\right) A_{i j}^{(1)} \otimes A_{k l}^{(2)}$, where $M=\left[m_{i j}^{i^{\prime} j^{\prime}}\right]$ and $N=\left[n_{k l}^{k^{\prime} l^{\prime}}\right]$ are two unitary matrices. Therefore, $S\left(\rho^{\prime}\right)=M^{t} S(\rho) N$. Let $\|P\|_{t r}$ stand for the trace norm of a matrix $P$ defined by $\|P\|_{t r}=\sum_{i} \sigma_{i}=\operatorname{tr} \sqrt{P^{\dagger} P}, P \in \mathbb{R}^{m \times n}$, where $\sigma_{i}$ $(i=1,2, \cdots, \min \{m, n\})$ are the singular values of the matrix $P$. Then we have $\left\|S\left(\rho^{\prime}\right)\right\|_{t r}=$ $\|S(\rho)\|_{t r}$ due to the fact that the singular values of a rectangular matrix $S$ are the same as those of $M^{t} S N$ when $M$ and $N$ are unitary matrices. Thus, the study of $\rho$ can be translated into that of $\rho^{\prime}$. Using this idea, we can simplify the matrices constructed by correlation tensors.

This paper is organized as follows. In Section 2, we derive the criteria to detect entanglement and GME of three-qubit states. By detailed examples, our results are seen to outperform some previously available results. In Section 3, we generalize the results to tripartite qudit quantum systems. Conclusions are given in Section 4.

\section{GME for three-qubit quantum states}

We first consider the GME for tripartite qubit states. Let $H_{i}^{d}$ denote the $i$-th $d$-dimensional Hilbert space. A tripartite pure state $|\varphi\rangle \in H_{1}^{d} \otimes H_{2}^{d} \otimes H_{3}^{d}$ is called biseparable under the bipartition $f \mid g h$ if $|\varphi\rangle$ can be written as $|\varphi\rangle=\left|\varphi_{f}\right\rangle \otimes\left|\varphi_{g h}\right\rangle$, where $\left|\varphi_{f}\right\rangle$ and $\left|\varphi_{g h}\right\rangle$ denote respectively the pure states in $H_{f}^{d}$ and $H_{g}^{d} \otimes H_{h}^{d}(f \neq g \neq h \in\{1,2,3\})$. Any non-biseparable pure state is said to be genuine multipartite entangled. A mixed state $\rho$ is called genuine multipartite entangled if it cannot be written as a convex combination of biseparable states.

For $d=2$, the principal basis matrices are given by

$$
A_{00}=\left[\begin{array}{ll}
1 & 0 \\
0 & 1
\end{array}\right], A_{01}=\left[\begin{array}{ll}
0 & 1 \\
1 & 0
\end{array}\right], A_{10}=\left[\begin{array}{cc}
1 & 0 \\
0 & -1
\end{array}\right], A_{11}=\left[\begin{array}{cc}
0 & 1 \\
-1 & 0
\end{array}\right]
$$


A general three-qubit state $\rho \in H_{1}^{2} \otimes H_{2}^{2} \otimes H_{3}^{2}$ can be expressed as,

$$
\begin{aligned}
\rho= & \frac{1}{8}\left(I_{2} \otimes I_{2} \otimes I_{2}+\sum_{(i, j)} u_{i j} A_{i j} \otimes I_{2} \otimes I_{2}+\sum_{(k, l)} v_{k l} I_{2} \otimes A_{k l} \otimes I_{2}+\sum_{(s, t)} w_{s t} I_{2} \otimes I_{2} \otimes A_{s t}\right. \\
& +\sum_{(i, j),(k, l)} x_{i j, k l} A_{i j} \otimes A_{k l} \otimes I_{2}+\sum_{(i, j),(s, t)} y_{i j, s t} A_{i j} \otimes I_{2} \otimes A_{s t}+\sum_{(k, l),(s, t)} z_{k l, s t} I_{2} \otimes A_{k l} \otimes A_{s t} \\
& \left.+\sum_{\substack{(i, j),(k, l),(s, t)}} r_{i j, k l, s t} A_{i j} \otimes A_{k l} \otimes A_{s t}\right),
\end{aligned}
$$

where $I_{2}$ denotes the 2 identity matrix, the summation indices in $(i, j)((k, l)$ and $(s, t))$ are not both zero, $r_{i j, k l, s t}=\operatorname{tr}\left(\rho A_{i j}^{\dagger} \otimes A_{k l}^{\dagger} \otimes A_{s t}^{\dagger}\right)$. Denote by $T_{1}^{1 \mid 23}, T_{2}^{1 \mid 23}, T_{3}^{1 \mid 23}, T_{1}^{2 \mid 13}, T_{2}^{2 \mid 13}, T_{3}^{2 \mid 13}, T_{1}^{3 \mid 12}$, $T_{2}^{3 \mid 12}$ and $T_{3}^{3 \mid 12}$ the matrices with entries $r_{01, k l, s t}, r_{10, k l, s t}, r_{11, k l, s t}, r_{i j, 01, s t}, r_{i j, 10, s t}, r_{i j, 11, s t}, r_{i j, k l, 01}$, $r_{i j, k l, 10}$ and $r_{i j, k l, 11}$, respectively. For example,

$$
T_{1}^{2 \mid 13}=\left[\begin{array}{ccc}
r_{01,01,01} & r_{01,01,10} & r_{01,01,11} \\
r_{10,01,01} & r_{10,01,10} & r_{10,01,11} \\
r_{11,01,01} & r_{11,01,10} & r_{11,01,11}
\end{array}\right]
$$

and the other matrices are arranged similarly. Set $S^{f \mid g h}=a_{f} T_{1}^{f \mid g h}+b_{f} T_{2}^{f \mid g h}+c_{f} T_{3}^{f \mid g h}$, where $a_{f}, b_{f}$ and $c_{f}$ are real constants, $f \neq g \neq h \in\{1,2,3\}$.

Let $\rho^{\prime}=\rho^{\left(I \otimes U_{2} \otimes U_{3}\right)}$, where $U_{2}, U_{3} \in U(2)$ are $2 \times 2$ unitary matrices. Assume $A_{i j}^{U_{2}}=$ $\sum_{\left(i^{\prime}, j^{\prime}\right) \neq(0,0)} m_{i j, i^{\prime} j^{\prime}} A_{i^{\prime} j^{\prime}}$ and $A_{i j}^{U_{3}}=\sum_{\left(i^{\prime}, j^{\prime}\right) \neq(0,0)} n_{i j, i^{\prime} j^{\prime}} A_{i^{\prime} j^{\prime}}$ with coefficient matrices $M=\left[m_{i j, i^{\prime} j^{\prime}}\right]$ and $N=\left[n_{i j, i^{\prime} j^{\prime}}\right]$. Then we have

$$
T_{1}^{1 \mid 23}\left(\rho^{\prime}\right)=M^{t} T_{1}^{1 \mid 23}(\rho) N, \quad T_{2}^{1 \mid 23}\left(\rho^{\prime}\right)=M^{t} T_{2}^{1 \mid 23}(\rho) N, \quad T_{3}^{1 \mid 23}\left(\rho^{\prime}\right)=M^{t} T_{3}^{1 \mid 23}(\rho) N .
$$

Therefore, $S^{1 \mid 23}\left(\rho^{\prime}\right)=M^{t} S^{1 \mid 23}(\rho) N$. Let $S^{1 \mid 23}(\rho)=U D V$ be the singular value decomposition of $S^{1 \mid 23}(\rho)$, where $U$ and $V$ are unitary matrices, $D$ is a diagonal matrix given by the singular values of $S^{1 \mid 23}(\rho)$. Then $S^{1 \mid 23}\left(\rho^{\prime}\right)=M^{t} U D V N$ has the same singular values as $S^{1 \mid 23}(\rho)$. Therefore, $\left\|S^{1 \mid 23}\left(\rho^{\prime}\right)\right\|_{t r}=\left\|S^{1 \mid 23}(\rho)\right\|_{t r}$. Namely, the trace norm is invariant under local unitary transformations.

Let us now consider the biseparable pure states. If $\rho=|\varphi\rangle\langle\varphi|$ is separable under the bipartition $1 \mid 23$, i.e., $|\varphi\rangle=\left|\varphi_{1}\right\rangle \otimes\left|\varphi_{23}\right\rangle \in H_{1}^{2} \otimes H_{23}^{4}$, using the Schmidt decomposition one has 
$|\varphi\rangle=t_{0}|0 \alpha\rangle+t_{1}|1 \beta\rangle$ under some suitable local bases, where $t_{0}^{2}+t_{1}^{2}=1$. Using LU equivalence, when $\left|\varphi_{23}\right\rangle \in H_{23}^{4}$ is separable we can transform $\{|\alpha\rangle,|\beta\rangle\}$ into two orthonormal bases which constitute separable states: (i) $\{|\alpha\rangle,|\beta\rangle\}=\{|00\rangle,|01\rangle\}$; when $\left|\varphi_{23}\right\rangle \in H_{23}^{4}$ is entangled, we can transform $\{|\alpha\rangle,|\beta\rangle\}$ into two orthonormal bases which constitute entangled states: (ii) $\{|\alpha\rangle,|\beta\rangle\}=\{|00\rangle,|11\rangle\}$. The matrices $T_{1}^{1 \mid 23}, T_{2}^{1 \mid 23}$ and $T_{3}^{1 \mid 23}$ are then given by

$$
\begin{gathered}
(i): T_{1}^{1 \mid 23}=\left[\begin{array}{ccc}
0 & 0 & 0 \\
2 t_{0} t_{1} & 0 & 0 \\
0 & 0 & 0
\end{array}\right], T_{2}^{1 \mid 23}=\left[\begin{array}{ccc}
0 & 0 & 0 \\
0 & t_{0}^{2}+t_{1}^{2} & 0 \\
0 & 0 & 0
\end{array}\right], T_{3}^{1 \mid 23}=\left[\begin{array}{ccc}
0 & 0 & 0 \\
0 & 0 & 2 t_{0} t_{1} \\
0 & 0 & 0
\end{array}\right] ; \\
(i i): T_{1}^{1 \mid 23}=\left[\begin{array}{ccc}
2 t_{0} t_{1} & 0 & 0 \\
0 & 0 & 0 \\
0 & 0 & 2 t_{0} t_{1}
\end{array}\right], T_{2}^{1 \mid 23}=\left[\begin{array}{ccc}
0 & 0 & 0 \\
0 & t_{0}^{2}-t_{1}^{2} & 0 \\
0 & 0 & 0
\end{array}\right], T_{3}^{1 \mid 23}=\left[\begin{array}{ccc}
0 & 0 & 2 t_{0} t_{1} \\
0 & 0 & 0 \\
2 t_{0} t_{1} & 0 & 0
\end{array}\right],
\end{gathered}
$$

corresponding to the cases (i) and (ii) respectively.

Lemma 1. For a biseparable pure state $\rho$, we have

(1) If $\rho$ is separable under the bipartition $1 \mid 23$, then $\left\|S^{1 \mid 23}\right\|_{t r}=\sqrt{4\left(a_{1}^{2}+c_{1}^{2}\right) t_{0}^{2} t_{1}^{2}+b_{1}^{2}}$ or $\left|b_{1}\right| \sqrt{\left(t_{0}^{2}-t_{1}^{2}\right)^{2}}+2 \mu_{1} t_{0} t_{1}$

(2) If $\rho$ is separable under the bipartition $2 \mid 13$, then $\left\|S^{2 \mid 13}\right\|_{t r}=\left|b_{2}\right|\left(1+4 t_{0} t_{1}\right)$ or $\left|b_{2}\right| \sqrt{\left(t_{0}^{2}-t_{1}^{2}\right)^{2}}+2 \mu_{2} t_{0} t_{1}$

(3) If $\rho$ is separable under the bipartition $3 \mid 12$, then $\left\|S^{3 \mid 12}\right\|_{t r}=\sqrt{4\left(a_{3}^{2}+c_{3}^{2}\right) t_{0}^{2} t_{1}^{2}+b_{3}^{2}}$ or $\left|b_{3}\right| \sqrt{\left(t_{0}^{2}-t_{1}^{2}\right)^{2}}+2 \mu_{3} t_{0} t_{1}$ corresponding to cases (i) or (ii), respectively, where $\mu_{f}=\left|a_{f}+c_{f}\right|+\left|a_{f}-c_{f}\right|$.

Proof. (1) By using the Schmidt decomposition and the expressions (4) and (5), the conclusion is easily seen.

(2) If a pure state $\rho=|\varphi\rangle\langle\varphi|$ is separable under $2 \mid 13$ bipartition, then $|\varphi\rangle=\left|\varphi_{2}\right\rangle \otimes\left|\varphi_{13}\right\rangle \in$ $H_{2}^{2} \otimes H_{13}^{4}$. Similarly we need to consider the following two cases: 
The first case: $\left|\varphi_{13}\right\rangle$ is separable. Under LU we have $|\varphi\rangle=t_{0}|000\rangle+t_{1}|101\rangle$, and

$$
T_{1}^{2 \mid 13}=\mathbf{0}, T_{2}^{2 \mid 13}=\left[\begin{array}{ccc}
2 t_{0} t_{1} & 0 & 0 \\
0 & t_{0}^{2}+t_{1}^{2} & 0 \\
0 & 0 & 2 t_{0} t_{1}
\end{array}\right], T_{3}^{2 \mid 13}=\mathbf{0}
$$

where $\mathbf{0}$ denotes the zero matrix. Then $\left\|S^{2 \mid 13}\right\|_{t r}=\left|b_{2}\right| \cdot\left[\left(t_{0}^{2}+t_{1}^{2}\right)+4 t_{0} t_{1}\right]=\left|b_{2}\right|\left(1+4 t_{0} t_{1}\right)$.

The second case: $\left|\varphi_{13}\right\rangle$ is entangled. Under LU we have $|\varphi\rangle=t_{0}|000\rangle+t_{1}|111\rangle$, and

$$
T_{1}^{2 \mid 13}=\left[\begin{array}{ccc}
2 t_{0} t_{1} & 0 & 0 \\
0 & 0 & 0 \\
0 & 0 & 2 t_{0} t_{1}
\end{array}\right], T_{2}^{2 \mid 13}=\left[\begin{array}{ccc}
0 & 0 & 0 \\
0 & t_{0}^{2}-t_{1}^{2} & 0 \\
0 & 0 & 0
\end{array}\right], T_{3}^{2 \mid 13}=\left[\begin{array}{ccc}
0 & 0 & 2 t_{0} t_{1} \\
0 & 0 & 0 \\
2 t_{0} t_{1} & 0 & 0
\end{array}\right] .
$$

We obtain $\left\|S^{2 \mid 13}\right\|_{t r}=\left|b_{2}\right| \sqrt{\left(t_{0}^{2}-t_{1}^{2}\right)^{2}}+2 \mu_{2} t_{0} t_{1}$.

(3) Since $S^{3 \mid 12}=\left(S^{1 \mid 23}\right)^{t}$ for the case (i) and $S^{3 \mid 12}=S^{1 \mid 23}$ for the case (ii), the conclusion can be proved in a similar way.

Remark There are two kinds of genuinely three-qubit entangled pure states under stochastic local operations and classical communication (SLOCC) [26], namely, the $G H Z$ state and $W$ state. We mix the GHZ state or W state with the white noise, by choosing different values of $\left\{a_{f}, b_{f}, c_{f}\right\}$, accordingly, we can obtain different upper bounds of $\left\|S^{f \mid g h}\right\|_{t r}$ to detect entanglement.

For mixed states, we have the following corollaries.

Corollary 1 For a quantum mixed state $\rho$, if $\left\|S^{2 \mid 13}\right\|_{t r}>3\left|b_{2}\right|$, where $3\left|b_{2}\right|<\mu_{2}\left(b_{2}, \mu_{2} \neq 0\right)$, then $\rho$ is entangled.

Corollary 2 For a quantum mixed state $\rho$, if $\left\|S^{f \mid g h}\right\|_{t r}=\left\|a_{f} T_{1}^{f \mid g h}+b_{f} T_{2}^{f \mid g h}\right\|_{t r}>\sqrt{b_{f}^{2}+4 a_{f}^{2}}$, where $\left|\frac{a_{f}}{b_{f}}\right|<1.6248$, then $\rho$ is entangled.

The proofs of the above corollaries can be seen respectively from the analysis of the following examples.

Example 1 Consider the mixed state $\rho_{G H Z}$,

$$
\rho_{G H Z}=\frac{x}{8} I_{8}+(1-x)|G H Z\rangle\langle G H Z|, 0 \leq x \leq 1
$$




\begin{tabular}{|c|c|}
\hline & the range of entanglement \\
\hline$a_{2}=-4, b_{2}=1, c_{2}=6$ & $0 \leq x<0.75$ \\
\hline$a_{2}=3, b_{2}=1, c_{2}=7$ & $0 \leq x<0.7857$ \\
\hline$a_{2}=5, b_{2}=\frac{1}{3}, c_{2}=5$ & $0 \leq x<0.9$ \\
\hline
\end{tabular}

Table 1: The entanglement ranges for $\rho_{G H Z}$ with respect to the the corresponding coefficients.

where $|G H Z\rangle=\frac{1}{\sqrt{2}}(|000\rangle+|111\rangle)$. We have $\left\|S^{2 \mid 13}\left(\rho_{G H Z}\right)\right\|_{t r}=\mu_{2}(1-x)$. By using Lemma 1 , when a mixed state $\rho=\sum p_{i} \rho_{i} \in H_{1}^{2} \otimes H_{2}^{2} \otimes H_{3}^{2}\left(0<p_{i} \leq 1, \sum p_{i}=1\right)$ is biseparable, we have

$$
\left\|S^{2 \mid 13}(\rho)\right\|_{t r} \leq \sum p_{i}\left\|S^{2 \mid 13}\left(\rho_{i}\right)\right\|_{t r} \leq\left|b_{2}\right|\left(1+4 t_{0} t_{1}\right) \leq 3\left|b_{2}\right| .
$$

Therefore, if $\rho_{G H Z}$ is separable under the bipartition $2 \mid 13$, we have $x \geq 1-\frac{3\left|b_{2}\right|}{\mu_{2}}$. Consequently, if $x<1-\frac{3\left|b_{2}\right|}{\mu_{2}}, \rho_{G H Z}$ is an entangled state. Since $x \in[0,1]$, we can detect the entanglement for $\rho_{G H Z}$ when $3\left|b_{2}\right|<\mu_{2}$. By using Corollary 1 , we get Table 1 of the entanglement ranges for $\rho_{G H Z}$. Obviously, these results are better than $0 \leq x<\frac{2}{3} \approx 0.6667$ given in [16].

Example 2 Consider the mixed state $\rho_{W}$,

$$
\rho_{W}=\frac{1-x}{8} I_{8}+x|W\rangle\langle W|, 0 \leq x \leq 1
$$

where $|W\rangle=\frac{1}{\sqrt{3}}(|100\rangle+|010\rangle+|001\rangle)$. When $c_{f}=0$ we have

$$
\begin{aligned}
\left\|S^{f \mid g h}\left(\rho_{W}\right)\right\|_{t r}= & \left\|a_{f} T_{1}^{f \mid g h}+b_{f} T_{2}^{f \mid g h}\right\|_{t r} \\
= & \frac{1}{\sqrt{18}}\left(\sqrt{8}+\sqrt{13+8\left(\frac{a_{f}}{b_{f}}\right)^{2}+\sqrt{25+16\left(\frac{a_{f}}{b_{f}}\right)^{2}}}\right. \\
& +\sqrt{\left.13+8\left(\frac{a_{f}}{b_{f}}\right)^{2}-\sqrt{25+16\left(\frac{a_{f}}{b_{f}}\right)^{2}}\right)\left|b_{f}\right| x .}
\end{aligned}
$$

By using Lemma 1, when a mixed state $\rho=\sum p_{i} \rho_{i} \in H_{1}^{2} \otimes H_{2}^{2} \otimes H_{3}^{2}\left(0<p_{i} \leq 1, \sum p_{i}=1\right)$ is biseparable, we have

$$
\left\|S^{f \mid g h}(\rho)\right\|_{t r} \leq \sum p_{i}\left\|S^{f \mid g h}\left(\rho_{i}\right)\right\|_{t r} \leq\left|b_{f}\right| \sqrt{\left(1-2 t_{1}^{2}\right)^{2}}+2 \mu_{f} t_{1} \sqrt{1-t_{1}^{2}} \leq \sqrt{b_{f}^{2}+\mu_{f}^{2}}
$$

where the upper bound is obtained by taking the extreme value of the function with independent variable $t_{1}$. If $\rho_{W}$ is separable under the bipartition $f \mid g h$, we have $\left\|S^{f \mid g h}\left(\rho_{W}\right)\right\|_{t r} \leq \sqrt{b_{f}^{2}+4 a_{f}^{2}}$. 


\begin{tabular}{|c|c|}
\hline & the range of entanglement \\
\hline$a_{f}=1, b_{f}=3$ & $0.5025<x \leq 1$ \\
\hline$a_{f}=1, b_{f}=10$ & $0.4361<x \leq 1$ \\
\hline$a_{f}=0, b_{f}=1$ & $0.4286<x \leq 1$ \\
\hline
\end{tabular}

Table 2: The entanglement ranges for $\rho_{W}$ with respect to the corresponding coefficients.

Consequently, if

$$
x>\frac{\sqrt{18} \sqrt{1+4\left(\frac{a_{f}}{b_{f}}\right)^{2}}}{\sqrt{8}+\sqrt{13+8\left(\frac{a_{f}}{b_{f}}\right)^{2}+\sqrt{25+16\left(\frac{a_{f}}{b_{f}}\right)^{2}}}+\sqrt{13+8\left(\frac{a_{f}}{b_{f}}\right)^{2}-\sqrt{25+16\left(\frac{a_{f}}{b_{f}}\right)^{2}}}}
$$

$\rho_{W}$ is an entangled state. The function $f(\delta)$ of $\delta=\frac{a_{f}}{b_{f}}$ at the right end of (13) is shown in Figure 1. Since $x \in[0,1]$, we can detect the entanglement for $\rho_{W}$ when $f(\delta)<1$, i.e., $|\delta|=\left|\frac{a_{f}}{b_{f}}\right|<1.6248$.

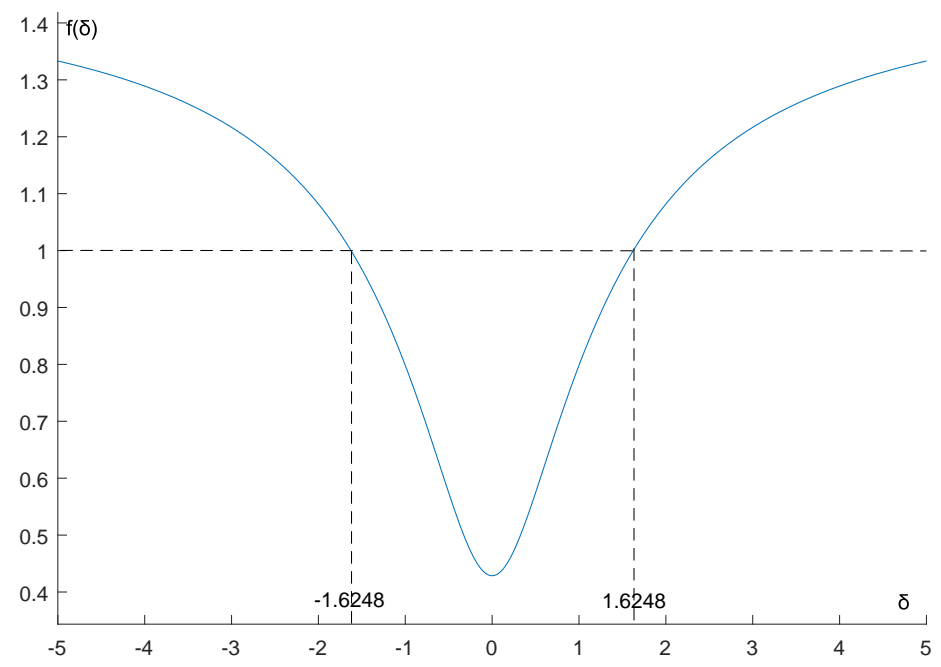

Figure 1: The image of function $f(\delta)$.

By using Corollary 2, we have Table 2 of entanglement ranges for $\rho_{W}$. These results are better than $0.6<x \leq 1$ that was given in [23]. Moreover, when $a_{f}=0$ and $b_{f}=1$, our criterion can detect more entanglement than $0.4334<x \leq 1$ which was given in [24]. 
We are now ready to derive the criterion of genuine multipartite entanglement. Set $T(\rho)=$ $\frac{1}{3}\left(\left\|S^{1 \mid 23}\right\|_{t r}+\left\|S^{2 \mid 13}\right\|_{t r}+\left\|S^{3 \mid 12}\right\|_{t r}\right)$. Using Lemma 1, we have the following theorem.

Theorem 1. For any mixed quantum state $\rho=\sum p_{i} \rho_{i} \in H_{1}^{2} \otimes H_{2}^{2} \otimes H_{3}^{2}\left(0<p_{i} \leq 1, \sum p_{i}=1\right)$, if $T(\rho)>M$, then $\rho$ is genuinely multipartite entangled, where $M=\max \left\{\sqrt{b_{1}^{2}+\mu_{1}^{2}}, \sqrt{b_{2}^{2}+\mu_{2}^{2}}\right.$, $\left.\sqrt{b_{3}^{2}+\mu_{3}^{2}}, 3\left|b_{2}\right|\right\}$.

Proof. For a pure state $\rho$, we have $T(\rho)=M_{1}$ or $M_{2}$ corresponding to the cases (i) or (ii), respectively, where

$$
\begin{gathered}
M_{1}=\frac{1}{3}\left[\sqrt{4\left(a_{1}^{2}+c_{1}^{2}\right) t_{0}^{2} t_{1}^{2}+b_{1}^{2}}+\left|b_{2}\right|\left(1+4 t_{0} t_{1}\right)+\sqrt{4\left(a_{3}^{2}+c_{3}^{2}\right) t_{0}^{2} t_{1}^{2}+b_{3}^{2}}\right], \\
M_{2}=\frac{1}{3} \sum_{f=1}^{3}\left(\left|b_{f}\right| \sqrt{\left(t_{0}^{2}-t_{1}^{2}\right)^{2}}+2 \mu_{f} t_{0} t_{1}\right) .
\end{gathered}
$$

Then for a mixed quantum state $\rho=\sum p_{i} \rho_{i}$,

$$
\begin{aligned}
T(\rho) & \leq \frac{1}{3} \sum p_{i}\left(\left\|S^{1 \mid 23}\left(\rho_{i}\right)\right\|_{t r}+\left\|S^{2 \mid 13}\left(\rho_{i}\right)\right\|_{t r}+\left\|S^{3 \mid 12}\left(\rho_{i}\right)\right\|_{t r}\right) \\
& \leq \max \left\{M_{1}, M_{2}\right\} \\
& \leq \max \left\{\sqrt{b_{1}^{2}+\mu_{1}^{2}}, \sqrt{b_{2}^{2}+\mu_{2}^{2}}, \sqrt{b_{3}^{2}+\mu_{3}^{2}}, 3\left|b_{2}\right|\right\} \\
& =M .
\end{aligned}
$$

Consequently, if $T(\rho)>M, \rho$ is a genuine multipartite entangled state.

Set $C=\left[\begin{array}{lll}a_{1} & b_{1} & c_{1} \\ a_{2} & b_{2} & c_{2} \\ a_{3} & b_{3} & c_{3}\end{array}\right]$. Our Theorem 1 can be used to detect GME by choosing different values of the entries of the matrix $C$.

Example 3 Consider the state $\rho_{W}$ defined in (10). With respect to different choices of $C$, we have the results showing in Table 3. From Lemma 2 in [15], $\rho$ has GME if $f(0, y)=$ $\frac{632 y^{2}}{9(3 y+1)}-10>0$, i.e., $0.6472<y \leq 1$. Set $h(x)=\frac{1}{12}(\sqrt{66} x-6)$. From Theorem 2 in [17], $\rho$ has GME if $h(x)>0$, i.e., $0.7385<x \leq 1$. From Table 3, our results show that $\rho$ has GME for $f(x)=54.4563 x-\sqrt{904}>0$, i.e., $0.5521<x \leq 1$. Therefore, our ranges are better than the results given in [15] and [17], see also Fig. 2. 


\begin{tabular}{|c|c|c|c|c|}
\hline & $\mathrm{C}$ & $T\left(\rho_{W}\right) \approx$ & M & the range of GME \\
\hline $\begin{array}{l}2 \\
0 \\
1\end{array}$ & $\begin{array}{ll}3 & 2 \\
\frac{5}{3} & 0 \\
4 & 1\end{array}$ & $7.0247 x$ & 5 & $0.7118<x \leq 1$ \\
\hline $\begin{array}{l}1 \\
0 \\
1\end{array}$ & $\begin{array}{cc}15 & 1 \\
5 & 0 \\
15 & 1\end{array}$ & $27.2459 x$ & $\sqrt{229}$ & $0.5554<x \leq 1$ \\
\hline $\begin{array}{l}1 \\
0 \\
1 \\
\end{array}$ & $\begin{array}{ll}3 & 1 \\
1 & 0 \\
3 & 1 \\
\end{array}$ & $5.5634 x$ & $\sqrt{13}$ & $0.6481<x \leq 1$ \\
\hline $\begin{array}{l}1 \\
0 \\
1\end{array}$ & $\begin{array}{ll}30 & 1 \\
10 & 0 \\
30 & 1\end{array}$ & $54.4563 x$ & $\sqrt{904}$ & $0.5521<x \leq 1$ \\
\hline
\end{tabular}

Table 3: $T\left(\rho_{W}\right), M$ and the range of GME of the state (10) for different $C$.

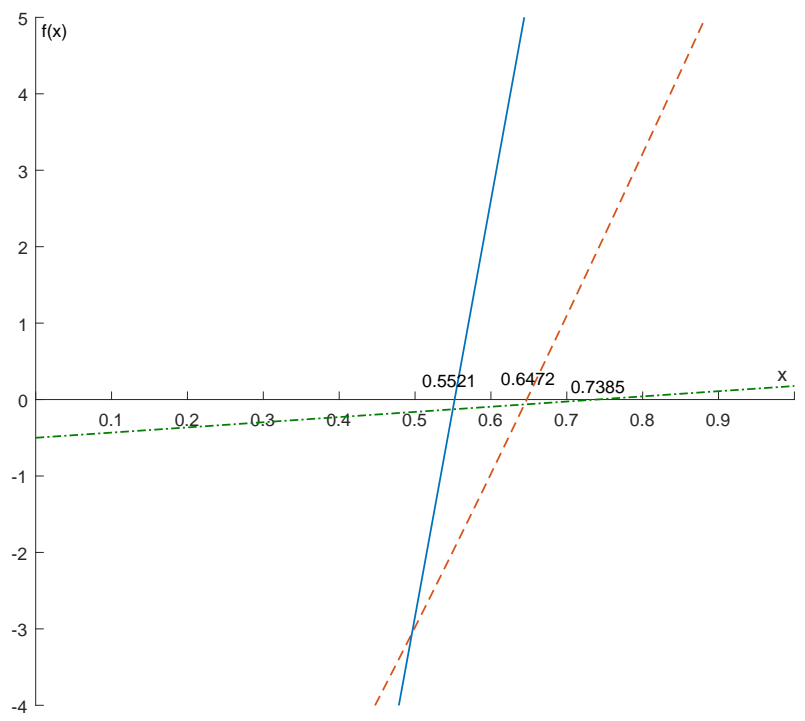

Figure 2: $f(x)$ from our result (solid straight line), $f(0, y)$ from Lemma 2 in [15] (dashed curve line), $h(x)$ from Theorem 2 in [17] (dash-dot straight line). 


\section{GME for tripartite qudit quantum states}

Next, we consider GME of $(d \times d \times d)$-dimensional quantum states. Different from the case of $d=2$ where all the principal basis matrices given in (1) are real, for $d \geq 3$ the principal basis matrices are complex. For instance, for $d=3$ the principal basis matrices are given by,

$$
\begin{aligned}
& A_{01}=\left[\begin{array}{lll}
0 & 1 & 0 \\
0 & 0 & 1 \\
1 & 0 & 0
\end{array}\right], A_{02}=\left[\begin{array}{lll}
0 & 0 & 1 \\
1 & 0 & 0 \\
0 & 1 & 0
\end{array}\right], A_{10}=\left[\begin{array}{lll}
1 & 0 & 0 \\
0 & \omega & 0 \\
0 & 0 & \omega^{2}
\end{array}\right], A_{11}=\left[\begin{array}{ccc}
0 & 1 & 0 \\
0 & 0 & \omega \\
\omega^{2} & 0 & 0
\end{array}\right], \\
& A_{12}=\left[\begin{array}{lll}
0 & 0 & 1 \\
\omega & 0 & 0 \\
0 & \omega^{2} & 0
\end{array}\right], A_{20}=\left[\begin{array}{lll}
1 & 0 & 0 \\
0 & \omega^{2} & 0 \\
0 & 0 & \omega
\end{array}\right], A_{21}=\left[\begin{array}{ccc}
0 & 1 & 0 \\
0 & 0 & \omega^{2} \\
\omega & 0 & 0
\end{array}\right], A_{22}=\left[\begin{array}{ccc}
0 & 0 & 1 \\
\omega^{2} & 0 & 0 \\
0 & \omega & 0
\end{array}\right]
\end{aligned}
$$

and $A_{00}=I_{3}$, where $\omega^{3}=1$. Therefore, we need to use different methods to deal with the GME problem.

For any state $\rho \in H_{1}^{d} \otimes H_{2}^{d} \otimes H_{3}^{d}(d \geq 3), \rho$ has the principal basis representation:

$$
\begin{aligned}
\rho= & \frac{1}{d^{3}}\left(I_{d} \otimes I_{d} \otimes I_{d}+\sum_{(i, j)} u_{i j} A_{i j} \otimes I_{d} \otimes I_{d}+\sum_{(k, l)} v_{k l} I_{d} \otimes A_{k l} \otimes I_{d}+\sum_{(s, t)} w_{s t} I_{d} \otimes I_{d} \otimes A_{s t}\right. \\
& +\sum_{(i, j),(k, l)} x_{i j, k l} A_{i j} \otimes A_{k l} \otimes I_{d}+\sum_{(i, j),(s, t)} y_{i j, s t} A_{i j} \otimes I_{d} \otimes A_{s t}+\sum_{(k, l),(s, t)} z_{k l, s t} I_{d} \otimes A_{k l} \otimes A_{s t} \\
& \left.+\sum_{\substack{(i, j),(k, l),(s, t)}} r_{i j, k l, s t} A_{i j} \otimes A_{k l} \otimes A_{s t}\right),
\end{aligned}
$$

where the summation indices in $(i, j),(k, l),(s, t)$ are not both simultaneously zero, $r_{i j, k l, s t}=$ $\operatorname{tr}\left(\rho A_{i j}^{\dagger} \otimes A_{k l}^{\dagger} \otimes A_{s t}^{\dagger}\right)$. Denote by $T_{i+1}^{1 \mid 23}, T_{k+1}^{2 \mid 13}$ and $T_{s+1}^{3 \mid 12}$ the matrices with entries $r_{i 1, k l, s t}, r_{i j, k 1, s t}$ 
and $r_{i j, k l, s 1}$, respectively, for example, when $d=4$,

$$
T_{3}^{1 \mid 23}=\left[\begin{array}{cccccc}
r_{21,01,01} & r_{21,01,02} & r_{21,01,03} & r_{21,01,10} & \cdots & r_{21,01,33} \\
r_{21,02,01} & r_{21,02,02} & r_{21,02,03} & r_{21,02,10} & \cdots & r_{21,02,33} \\
r_{21,03,01} & r_{21,03,02} & \cdot & \cdot & \cdots & \cdot \\
r_{21,10,01} & r_{21,10,02} & \cdot & . & \cdots & \cdot \\
\vdots & \vdots & \vdots & \vdots & & \vdots \\
r_{21,13,01} & r_{21,13,02} & . & . & \cdots & \cdot \\
\vdots & \vdots & \vdots & \vdots & \vdots & \vdots \\
r_{21,30,01} & r_{21,30,02} & . & . & \cdots & \cdot \\
\vdots & \vdots & \vdots & \vdots & & \vdots \\
r_{21,33,01} & r_{21,33,02} & . & . & \cdots & .
\end{array}\right]
$$

the other matrices are arranged in a similar way. Set $N^{1 \mid 23}=\sum_{i} T_{i+1}^{1 \mid 23}, N^{2 \mid 13}=\sum_{k} \omega^{k} T_{k+1}^{2 \mid 13}$, $N^{3 \mid 12}=\sum_{s} \omega^{s} T_{s+1}^{3 \mid 12}$ and $T(\rho)=\frac{1}{3}\left(\left\|N^{1 \mid 23}\right\|_{t r}+\left\|N^{2 \mid 13}\right\|_{t r}+\left\|N^{3 \mid 12}\right\|_{t r}\right)$, where $i, j, k, l, s, t \in Z_{d}$.

Lemma 2. For a pure state $\rho \in H_{1}^{d} \otimes H_{2}^{d} \otimes H_{3}^{d}(d \geq 3)$, we have

(1) If $\rho$ is separable under bipartition $1 \mid 23$, then $\left\|N^{1 \mid 23}\right\|_{t r} \leq \frac{\sqrt{d^{3}(d-1)}}{2}$ or $\frac{d^{2}}{2}$;

(2) If $\rho$ is separable under bipartition $2 \mid 13$, then $\left\|N^{2 \mid 13}\right\|_{t r} \leq \frac{d^{2}}{2}$;

(3) If $\rho$ is separable under bipartition $3 \mid 12$, then $\left\|N^{3 \mid 12}\right\|_{t r} \leq \frac{\sqrt{d^{3}(d-1)}}{2}$ or $\frac{d^{2}}{2}$.

Proof. (1) If $\rho$ is separable under bipartition $1 \mid 23$, we have $\left|\varphi_{1 \mid 23}\right\rangle=\left|\varphi_{1}\right\rangle \otimes\left|\varphi_{23}\right\rangle \in H_{1}^{d} \otimes H_{23}^{d^{2}}$, where $H_{23}^{d^{2}}=H_{2}^{d} \otimes H_{3}^{d}$. It follows from the Schmidt decomposition that $\left|\varphi_{1 \mid 23}\right\rangle=t_{0}\left|0 \alpha_{0}\right\rangle+$ $t_{1}\left|1 \alpha_{1}\right\rangle \cdots+t_{d-1}\left|d-1, \alpha_{d-1}\right\rangle$, where $\sum t_{i}^{2}=1$. Taking into account local unitary equivalence in $H_{2}^{d} \otimes H_{3}^{d}$, if $\left|\varphi_{23}\right\rangle \in H_{23}^{d^{2}}$ is separable, we can transform $\left\{\left|\alpha_{0}\right\rangle,\left|\alpha_{1}\right\rangle, \cdots,\left|\alpha_{d-1}\right\rangle\right\}$ into orthonormal bases which constitute separable states: (i) $\left\{\left|\alpha_{0}\right\rangle,\left|\alpha_{1}\right\rangle, \cdots,\left|\alpha_{d-1}\right\rangle\right\}=\{|00\rangle,|01\rangle, \cdots, \mid 0, d-$ $1\rangle$ \}, i.e., $\left|\varphi_{1 \mid 23}\right\rangle=\left|\varphi_{1}\right\rangle \otimes\left|\varphi_{2}\right\rangle \otimes\left|\varphi_{3}\right\rangle$ is fully separable. If $\left|\varphi_{23}\right\rangle \in H_{23}^{d^{2}}$ is entangled, we can transform $\left\{\left|\alpha_{0}\right\rangle,\left|\alpha_{1}\right\rangle, \cdots,\left|\alpha_{d-1}\right\rangle\right\}$ into orthonormal bases which constitute entangled states: (ii) $\left\{\left|\alpha_{0}\right\rangle,\left|\alpha_{1}\right\rangle, \cdots,\left|\alpha_{d-1}\right\rangle\right\}=\{|00\rangle,|11\rangle, \cdots,|d-1, d-1\rangle\}$.

For the first case $(\mathrm{i}),|\varphi\rangle=|000\rangle+|101\rangle+\cdots+|d-1,0, d-1\rangle$, we have $N^{1 \mid 23}=$ $d t_{0} t_{1}|\gamma\rangle\langle\eta|$, where $|\gamma\rangle=\left[\begin{array}{lllllllll}0 & \cdots & 1_{(1, d)} & \cdots & 1_{(1,2 d)} & \cdots & 1_{(1,(d-1) d)} & \cdots & 0\end{array}\right]^{t}$, and $|\eta\rangle=$ $\left[\begin{array}{lllllll}1_{(1,1)} & \cdots & 1_{(1, d+1)} & \cdots & 1_{(1,(d-1) d+1)} & \cdots & 0\end{array}\right]^{t}, a_{(i, j)}$ denotes that the element $a$ is located at 
the $i$ th row and $j$ th column of the matrix. Therefore, $\left\|N^{1 \mid 23}\right\|_{t r}=d t_{0} t_{1} \sqrt{d(d-1)} \leq \frac{\sqrt{d^{3}(d-1)}}{2}$, where we have used $\left.\||\gamma\rangle\left\langle\eta\left|\left\|_{t r}=\right\|\right| \gamma\right\rangle\|\| \| \eta\right\rangle \|$ for vectors $|\gamma\rangle$ and $|\eta\rangle$. For the second case (ii), $|\varphi\rangle=|000\rangle+|111\rangle+\cdots+|d-1, d-1, d-1\rangle$, we have $N^{1 \mid 23}=d t_{0} t_{1}|\eta\rangle\langle\eta|$. Therefore, $\left\|N^{1 \mid 23}\right\|_{t r}=d t_{0} t_{1} \sqrt{d^{2}} \leq \frac{d^{2}}{2}$.

(2) If $\rho$ is separable under bipartition 2|13, we need to consider again two cases. For the first case, $|\varphi\rangle=|000\rangle+|101\rangle+\cdots+|d-1,0, d-1\rangle$, and we have $T_{k+1}^{2 \mid 13}=\mathbf{0}\left(k \in Z_{d}\right)$, where $\mathbf{0}$ is the zero matrix. Therefore, $\left\|N^{2 \mid 13}\right\|_{t r}=0$. For the second case, $|\varphi\rangle=|000\rangle+|111\rangle+\cdots+\mid d-1, d-1, d-$ $1\rangle$, one has $N^{2 \mid 13}=d t_{1} t_{2}|\xi\rangle\langle\xi|$, where $|\xi\rangle=\left[\begin{array}{lllllll}1_{(1,1)} & \cdots & \bar{\omega}_{(1, d+1)} & \cdots & \cdots & \bar{\omega}^{d-1} & \\ (1,(d-1) d+1) & \cdots & 0\end{array}\right]^{t}$, where $\bar{\omega}$ denotes the complex conjugate of $\omega$. Then we have $\left\|N^{2 \mid 13}\right\|_{t r}=d^{2} t_{1} t_{2} \leq \frac{d^{2}}{2}$.

(3) If $\rho$ is separable under bipartition $3 \mid 12$, for the first case, $N^{3 \mid 12}=d t_{1} t_{2}|\xi\rangle\langle\gamma|$. Then we have $\left\|N^{3 \mid 12}\right\|_{t r}=d t_{1} t_{2} \sqrt{d(d-1)} \leq \frac{\sqrt{d^{3}(d-1)}}{2}$. For the second case, $N^{3 \mid 12}=d t_{1} t_{2}|\xi\rangle\langle\xi|$, we have $\left\|N^{3 \mid 12}\right\|_{t r}=d^{2} t_{1} t_{2} \leq \frac{d^{2}}{2}$.

Theorem 2. For any mixed quantum state $\rho=\sum p_{i} \rho_{i} \in H_{1}^{d} \otimes H_{2}^{d} \otimes H_{3}^{d}\left(0<p_{i} \leq 1, \sum p_{i}=\right.$ $1, d \geq 3)$, if $T(\rho)>\frac{d^{2}}{2}$, then $\rho$ is genuine multipartite entangled.

Proof. If $\rho$ is biseparable, then by Lemma 2 we have

$$
T(\rho) \leq \frac{1}{3} \sum p_{i}\left(\left\|N^{1 \mid 23}\left(\rho_{i}\right)\right\|_{t r}+\left\|N^{2 \mid 13}\left(\rho_{i}\right)\right\|_{t r}+\left\|N^{3 \mid 12}\left(\rho_{i}\right)\right\|_{t r}\right) \leq \frac{d^{2}}{2}
$$

Consequently, if $T(\rho)>\frac{d^{2}}{2}, \rho$ is genuine mulitipartite entangled.

\section{Conclusion}

We have studied the entanglement and genuine multipartite entanglement in tripartite quantum systems. Based on the principal matrix representation, we constructed special matrices from the correlation tensors of the tripartite qubit states and have shown that they are invariant under local unitary transformation and are capable to detect quantum entanglement. From different linear combinations of these matrices, we have obtained new separability criteria and conditions on genuine multipartite entanglement. It has been shown that our criteria are 
more effective than some existing ones. By employing different methods for high dimensional cases, we have also studied the GME in $(d \times d \times d)$-dimensional quantum systems.

Acknowledgements This work is supported by the National Natural Science Foundation of China under grant nos. 11101017, 11531004, 11726016, 12075159, 12126351 and 12171044 , Simons Foundation under grant no. 523868, Beijing Natural Science Foundation (Z190005), Academy for Multidisciplinary Studies, Capital Normal University, the Academician Innovation Platform of Hainan Province, and Shenzhen Institute for Quantum Science and Engineering, Southern University of Science and Technology (no. SIQSE202001).

\section{References}

[1] Ekert, A.K.: Quantum cryptography based on Bell's theorem. Phys. Rev. Lett. 67, 661 (1991)

[2] Bennett, C.H., Brassard, G., Jozsa, R. et al: Teleporting an unknown quantum state via dual classical and Einstein-Podolsky-Rosen channels. Phys. Rev. Lett. 70, 1895 (1993)

[3] Bennett, C.H., Wiesner, S.J.: Communication via One-and Two-Particle operators on EinsteinPodolsky-Rosen states. Phys. Rev. Lett. 69, 2881 (1992)

[4] Hyllus, P., Laskowski, W., Krischek, R. et al: Fisher information and multiparticle entanglement. Phys. Rev. A 85, 022321 (2012)

[5] Tóth, G.: Multipartite entanglement and high precision metrology. Phys. Rev. A 85, 022322 (2012)

[6] Hong, Y., Gao, T., Yan, F. L.: Detection of k-partite entanglement and k-nonseparability of multipartite quantum states. Phys. Lett. A 401, 127347 (2021)

[7] Liu, L., Gao, T., Yan, F.: Separability criteria via some classes of measurements. Sci. China Phys. Mech. Astron. 60, 100311 (2017)

[8] Gao T., Hong Y., Lu, Y. et al: Efficient k-separability criteria for mixed multipartite quantum states. Europhysics Letters 104, 20007 (2013)

[9] Hong, Y., Qi, X. F., Gao, T. et al: Detection of multipartite entanglement via quantum Fisher information. Europhysics Letters 134, 60006 (2021)

[10] Gao, T., Yan, F. L., Van Enk S. J.: Permutationally invariant part of a density matrix and nonseparability of n-qubit states. Phys. Rev. Lett. 112, 180501 (2014) 
[11] Gao T., Hong Y.: Detection of genuinely entangled and nonseparable n-partite quantum states. Phys. Rev. A 82, 062113 (2010)

[12] Teo, Y. S., Struchalin, G. I., Kovlakov, E. V. et al: Objective compressive quantum process tomography. Phys. Rev. A 101, 022334 (2020)

[13] Tóth G., Gühne O.: Detecting genuine multipartite entanglement with two local measurements. Phys. Rev. Lett. 94, 060501 (2005)

[14] Li, J., Chen, L.: Detection of genuine multipartite entanglement based on uncertainty relations. Quantum Inf. Process. 20, 220 (2021)

[15] Yang, L. M., Sun, B. Z., Chen, B. et al: Quantum Fisher information-based detection of genuine tripartite entanglement. Quantum Inf. Process. 19, 262 (2020)

[16] Akbari-kourbolagh, Y.: Entanglement criteria for the three-qubit states. Int. J. Quantum Inf. 15, $1750049(2017)$

[17] Li, M., Jia, L.X., Wang, J. et al: Measure and detection of genuine multipartite entanglement for tripartite systems. Phys. Rev. A 96, 052314 (2017)

[18] Li, M., Fei, S. M., Wang, Z. X.: Separability of tripartite quantum systems. Int. J. Quantum Inf. 6, 859 (2008)

[19] Shen, S. Q., Yu, J., Li, M.:Improved separability criteria based on Bloch representation of density matrices. Sci. Rep. 6, 28850 (2016)

[20] Liu, M., Bai, C. M., Ge, M.-L., Jing, N.: Generalized Bell states and principal realization of the Yangian $Y(\mathfrak{s l}(N)$. J. Math. Phys. 54, 021701, (2013).

[21] Huang, X. F., Jing, N., Zhang, T. G.: An upper bound of fully entangled fraction of mixed states. Commun. in Theor. Phys. 65, 701 (2016)

[22] Nielsen, M. A., Chuang, I. L.: Quantum computation and quantum information. Cambridge Univ. Press, Cambridge, (2000)

[23] Acín, A., Bruß, D., Lewenstein, M., Sanpera, A.: Classification of mixed three-qubit states. Phys. Rev. Lett. 87, 040401 (2001)

[24] Akbari-Kourbolagh, Y., Azhdargalam, M.: Entanglement criterion for tripartite systems based on local sum uncertainty relations. Phys. Rev. Lett. 97, 042333 (2018)

[25] Jing, N., Yang, M., Zhao, H.:Local unitary equivalence of quantum states and simultaneous orthogonal equivalence. J. Math. Phys. 57, 062205 (2016) 
[26] Dür, W., Vidal, G., Cirac, J. I.:Three qubits can be entangled in two inequivalent ways. Phys. Rev. A 62, 062314 (2000) 